\title{
Advancements in the Music Information Retrieval Framework AMUSE over the Last Decade
}

\author{
Igor Vatolkin \\ igor.vatolkin@tu-dortmund.de \\ Department of Computer Science \\ TU Dortmund University \\ Germany
}

\author{
Philipp Ginsel \\ philipp.ginsel@tu-dortmund.de \\ Department of Computer Science \\ TU Dortmund University \\ Germany
}

\author{
Günter Rudolph \\ guenter.rudolph@tu-dortmund.de \\ Department of Computer Science \\ TU Dortmund University \\ Germany
}

\begin{abstract}
AMUSE (Advanced MUSic Explorer) was created 2006 as an opensource Java framework for various music information retrieval tasks like feature extraction, feature processing, classification, and evaluation. In contrast to toolboxes which focus on individual MIR-related algorithms, it is possible with AMUSE, for instance, to extract features with Librosa, process them based on events estimated by MIRtoolbox, classify with WEKA or Keras, and validate the models with own classification performance measures. We present several substantial contributions to AMUSE since its first presentation at ISMIR 2010. They include the annotation editor for single and multiple tracks, the support of multi-label and multi-class classification, and new plugins which operate with Keras, Librosa, and Sonic Annotator. Other integrated methods are the structural complexity processing, chord vector feature, aggregation of features around estimated onset events, and evaluation of time event extractors. Further advancements are a more flexible feature extraction with different parameters like frame sizes, possibility to integrate additional tasks beyond algorithms related to supervised classification, marking of features which can be ignored for a classification task, extension of algorithm parameters with external code (e.g., a structure of a Keras neural net), etc.
\end{abstract}

\section{CCS CONCEPTS}

- Information systems $\rightarrow$ Music retrieval; • Applied computing $\rightarrow$ Sound and music computing; • Computing methodologies $\rightarrow$ Machine learning approaches.

\section{KEYWORDS}

music information retrieval, music data analysis, music classification, audio feature extraction, evaluation of music classification

\section{ACM Reference Format:}

Igor Vatolkin, Philipp Ginsel, and Günter Rudolph. 2021. Advancements in the Music Information Retrieval Framework AMUSE over the Last Decade. In Proceedings of the 44th International ACM SIGIR Conference on Research and Development in Information Retrieval (SIGIR '21), July 11-15, 2021, Virtual Event, Canada. ACM, New York, NY, USA, 7 pages. https://doi.org/10.1145/ 3404835.3463252

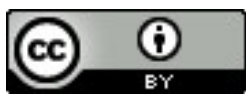

This work is licensed under a Creative Commons Attribution International 4.0 License. SIGIR '21, July 11-15, 2021, Virtual Event, Canada. (C) 2021 Copyright held by the owner/author(s). ACM ISBN 978-1-4503-8037-9/21/07.

https://doi.org/10.1145/3404835.3463252

\section{INTRODUCTION}

The motivation to create the open-source Java framework AMUSE (Advanced MUSic Explorer) [25] in 2006 was that for different subtasks in the supervised music classification chain, with a particular focus on music genre and style recognition, it was often necessary to use several tools. Some were mainly developed for rather specific tasks such as audio signal feature extraction, like jAudio [10] or MIRtoolbox [8]. Others were more complex frameworks for various classification and data processing tasks, like WEKA [3] or YALE [13] (former name of RapidMiner [6]), which were overburdened with many algorithms not related to Music Information Retrieval (MIR). The integration of feature processing methods based on music time events (onsets, beats, etc.) required own implementations. Even if the existing toolboxes shared the same input/output format, the results could be differently organized: e.g., the two dimensions of the feature matrix, the feature vector components and the extraction time frames, were stored either as rows or columns. Further, for a more robust evaluation and optimization of MIR applications, it was required to implement a large set of different evaluation criteria, and also to enable a parallel handling of subtasks for computing on grid systems. Thus, a framework with a standardized task definition, well-defined input/output formats, support of plugins and simple extensions, and evaluation routines would substantially help to test, compare, and optimize different MIR algorithms.

Many things have changed since that time. Deep neural networks have been established as the first choice for many music classification tasks. A lot of machine learning and audio processing algorithms have moved to and been implemented in Python modules. But also many new methods were integrated into general data mining frameworks with a long history like MATLAB or RapidMiner. However, there is still a lack of software systems which on one side are focused and specialized on MIR and on the other side address a variety of different related tasks allowing for the communication with other toolboxes or frameworks.

In this paper, we provide a summary of the most significant advancements and changes in AMUSE over the last decade after its first presentation at ISMIR 2010. In Section 2, we start with an overview of AMUSE basic tasks. In Section 3, we list the changes in AMUSE nodes (software components responsible for the related individual tasks like feature extraction and classification). Section 4 introduces the annotation editor which helps to create ground truth for music classification experiments. Section 5 outlines further advancements. Section 6 describes research and teaching experiences with AMUSE. Section 7 discusses several ideas for future work.

AMUSE is publicly available on the GitHub website: https:/gith ub.com/AdvancedMUSicExplorer/AMUSE. 


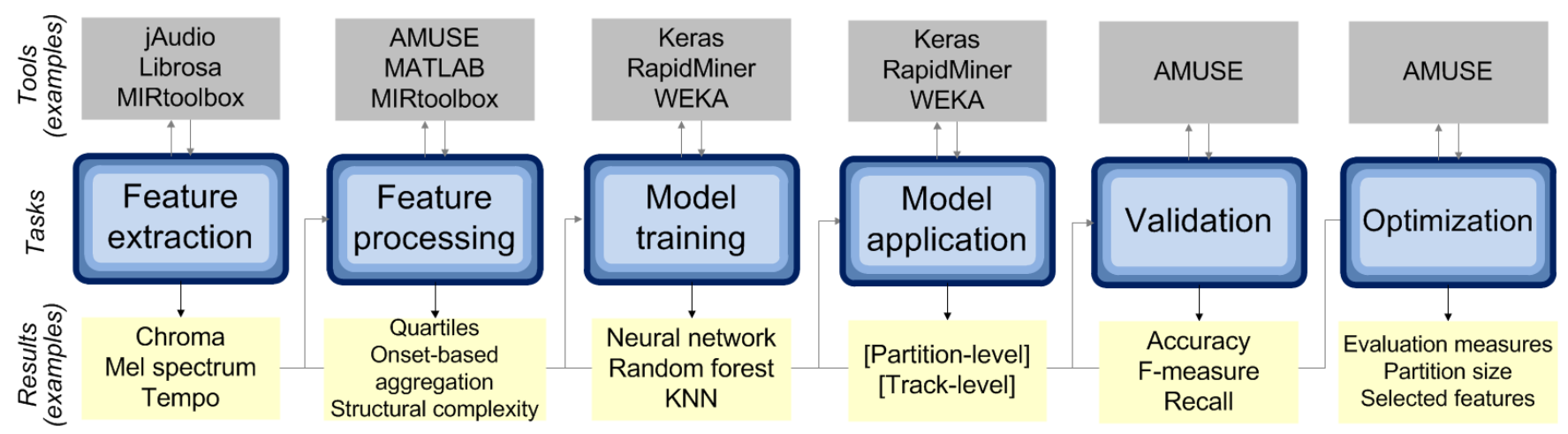

Figure 1: Overview of basic AMUSE tasks.

\section{TASK OVERVIEW}

Figure 1 shows the list of common subtasks in a supervised classification chain. Often the first step, the feature extraction stores some numeric or nominal properties of music data from different domains. For the audio signal, the most common ones are the time signal, the spectrum, and the cepstrum. Then, the features are usually processed with respect to feature and time dimensions. For example, they can be normalized to a given range, the number of feature dimensions can be reduced by means of principal component analysis or feature selection, or feature statistics like quartiles or coefficients of autoregressive models can be estimated for larger analysis frames. Other processing methods can even increase the dimensionality, adding, e.g., derivations or running average to the original feature time series. In general, the task of feature processing is to create feature vectors which represent classification instances (or windows, if, e.g., a segment of a music track should be classified) and are either supported with annotations to train supervised classification models or can be used to apply previously trained models or create unsupervised classification models.

Thus, in the next step, models are trained to organize music data either in an unsupervised way (e.g., assigning them to clusters) or to classify music data into different categories: genres, emotions, instruments, chords, harmonic progressions, etc. After the models are saved, they can be later applied to categorize new data. The validation task measures the performance of classification models, but can be applied also for the estimation of further criteria, like computing time, storage space, or the size of the training set. Here, different strategies like cross-validation can be applied for a systematic and robust evaluation of models. The objects to classify can be of different lengths: from small segments of music pieces (e.g., for the prediction of vocal occurrence or chords) to complete music tracks for the prediction of music genres and styles. In the optimization task, the algorithms can be tuned to perform as good as possible with respect to selected criteria.

Each task is managed by the corresponding AMUSE "node" which configures and starts either methods implemented in AMUSE itself or communicates with other tools which may be implemented in Java, MATLAB, Python, or any other programming language if they can be started via command line. Tools which do not belong to the main AMUSE framework and are not integrated as Java JAR libraries, can be installed and removed as plugins. The results are stored in AMUSE workspace either as WEKA ARFF [4] files which can be easily read and manually edited (extracted and processed features, model predictions, evaluation measures, and optimization logs) or as binary files (classification models).

The configuration of AMUSE experiments is done with the help of ARFF files or also using a graphical user interface with an experiment table as visualized in Figure 2 with the following markers:

\begin{tabular}{l}
1 : Selection of an experiment from the six available tasks. \\
\hline 2 : After the setup of experiment parameters, it appears in \\
the table, showing some general details and the current state \\
of the experiment (CONFIGURED, IN_QUEUE, RUNNING, \\
FINISHED). \\
3 : Configured experiments can be stored or the previously \\
configured experiments can be loaded. \\
4 : By pressing "Start Experiments" button, the experiments \\
are started.
\end{tabular}

\section{CHANGES IN AMUSE NODES}

In the following, we list the most important changes in AMUSE nodes from the feature extraction to validation.

\subsection{Feature Extraction}

It is now distinguished between features of five types: WindowedNumeric, WindowedString, Event, SegmentedNumeric, SegmentedString. Windowed*-features are extracted from the frames with the same length and step size measured in samples. Event features are individual time points (e.g., onsets or beat times). Segmented*features describe segments of music tracks or can be also used to describe chord progressions or other features with variable extraction frame sizes. Each Windowed*- or Segmented ${ }^{*}$-feature can have a numeric or nominal value.

To provide a more flexible feature extraction, it is now possible to define several configurations for the same feature to extract. For instance, the standard MFCC feature vector is extracted from a 512-sample frame with a 512-sample step size. Now, it is possible to define an additional configuration for MFCCs extracted from a 1024-sample frame, estimating a larger number of dimensions, or even using another implementation or tool. The corresponding 


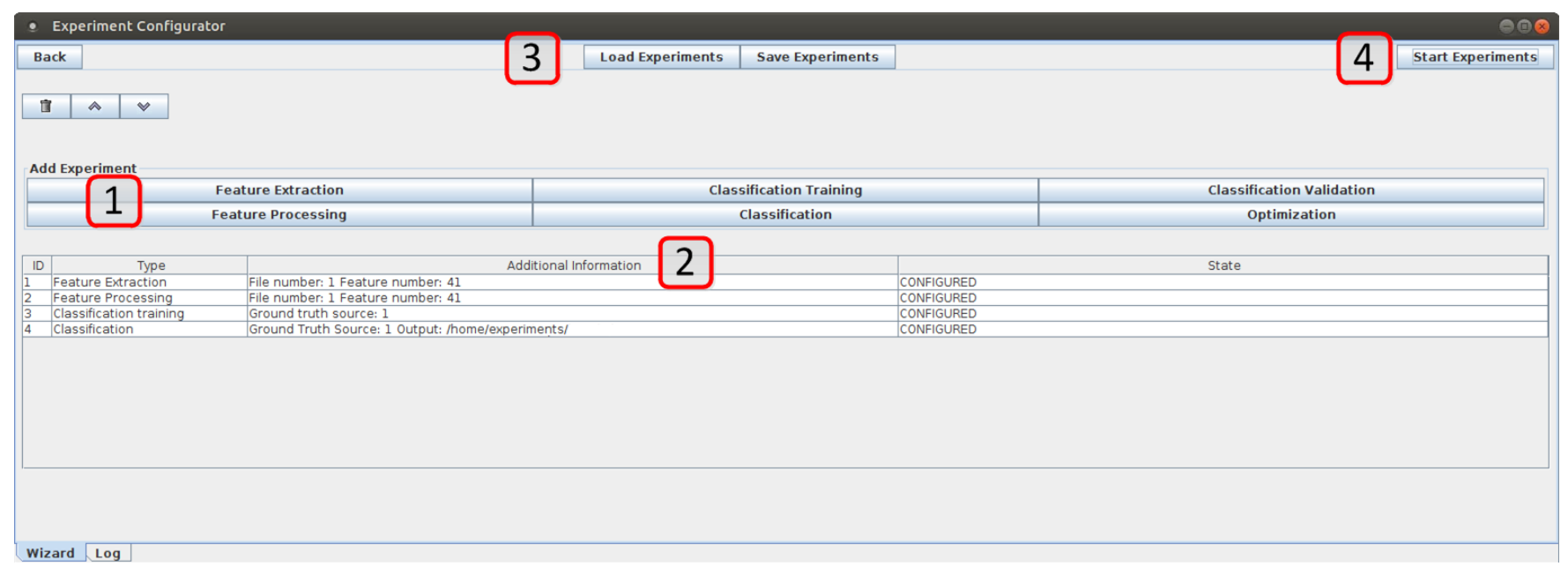

Figure 2: Screenshot with experiment configuration table.

features are stored in the format [MusicFile]_[FeatureID]_[Additi onalConfigurationID].arff.

Plugins which operate with Librosa [11] (https://github.com/Adv ancedMUSicExplorer/AMUSE/tree/master/amusePluginLibrosa) and Sonic Annotator [1] (https://github.com/AdvancedMUSicEx plorer/AMUSE/tree/master/amusePluginSonicAnnotator) were added.

Several features were added, among others:

- Chroma DCT-Reduced log Pitch (CRP) [14] available in ChromaToolbox [15] AMUSE plugin (https://github.com/Advan cedMUSicExplorer/AMUSE/tree/master/amusePluginChro maToolbox),

- the strengths of all possible intervals based on CRP, numbers of different chords, their changes, the shares of the most frequent chords,

- the new chord representation [5] available in Sonic Annotator plugin,

- the Mel spectrum available in the Librosa plugin, and

- the extractor of segment boundaries based on novelty function and the initial implementation of [16] extended in [21].

\subsection{Feature Processing}

Several algorithms were added to feature processing, like the normalization to zero mean and unit standard deviation and the raw feature processor, which only aggregates features for given classification windows that can be used, e.g., as input to the training of neural networks. The original continuous feature values can be discretized to a limited set of values based on percentile analysis or histograms. The temporal progress of feature groups related to some musically meaningful property like harmony, chords, or tempo, can be measured as the structural complexity following [9]. The features can be aggregated not only for classification frames of a given length, but also around previously extracted onset events. In contrast to earlier AMUSE versions, it is now possible to apply feature processing task not only to the original previously extracted features, but also to the already processed features which are stored in another ARFF format.

\subsection{Classification}

In the former versions of AMUSE, it was only possible to apply binary supervised classification models. Now, model types can be selected with respect to the following properties:

- Relationship Type: BINARY or CONTINUOUS. Continuous outputs contain relationship values $y_{i} \in[0,1], y_{i} \in \mathbb{R}$ to one or several categories and are used, for instance, for fuzzy classification algorithms based on fuzzy set theory with uncertain predictions [26].

- Label Type: SINGLE-LABEL, MULTI-CLASS, and MULTILABEL. Single-label algorithms assign instances to either "positive" or "negative" labels of a given category, e.g., "a segment with vocals" or "a segment without vocals". Note that these predictions must not be binary and can consider uncertainty. Multi-class algorithms assign instances to a single label selected from more than two possible categories. Multi-label algorithms assign instances to more than one label, for example, when a music track belongs to several genres.

- Method Type: Currently, only SUPERVISED is supported (models trained with ground truth annotations of labeled music data instances), but UNSUPERVISED type is planned for near future (models which do not need ground truth, like clustering or self-organized maps). Later, we plan also to support regression methods.

It is now possible to mark individual feature dimensions in a processed feature set saved as ARFF file which should not be taken into account during the training or classification.

A Keras [2] plugin (https://github.com/AdvancedMUSicExpl orer/AMUSE/tree/master/amusePluginKeras) was added which allows for the classification with deep neural networks. Python and Keras together with further required modules must be installed separately and are not distributed within AMUSE. 


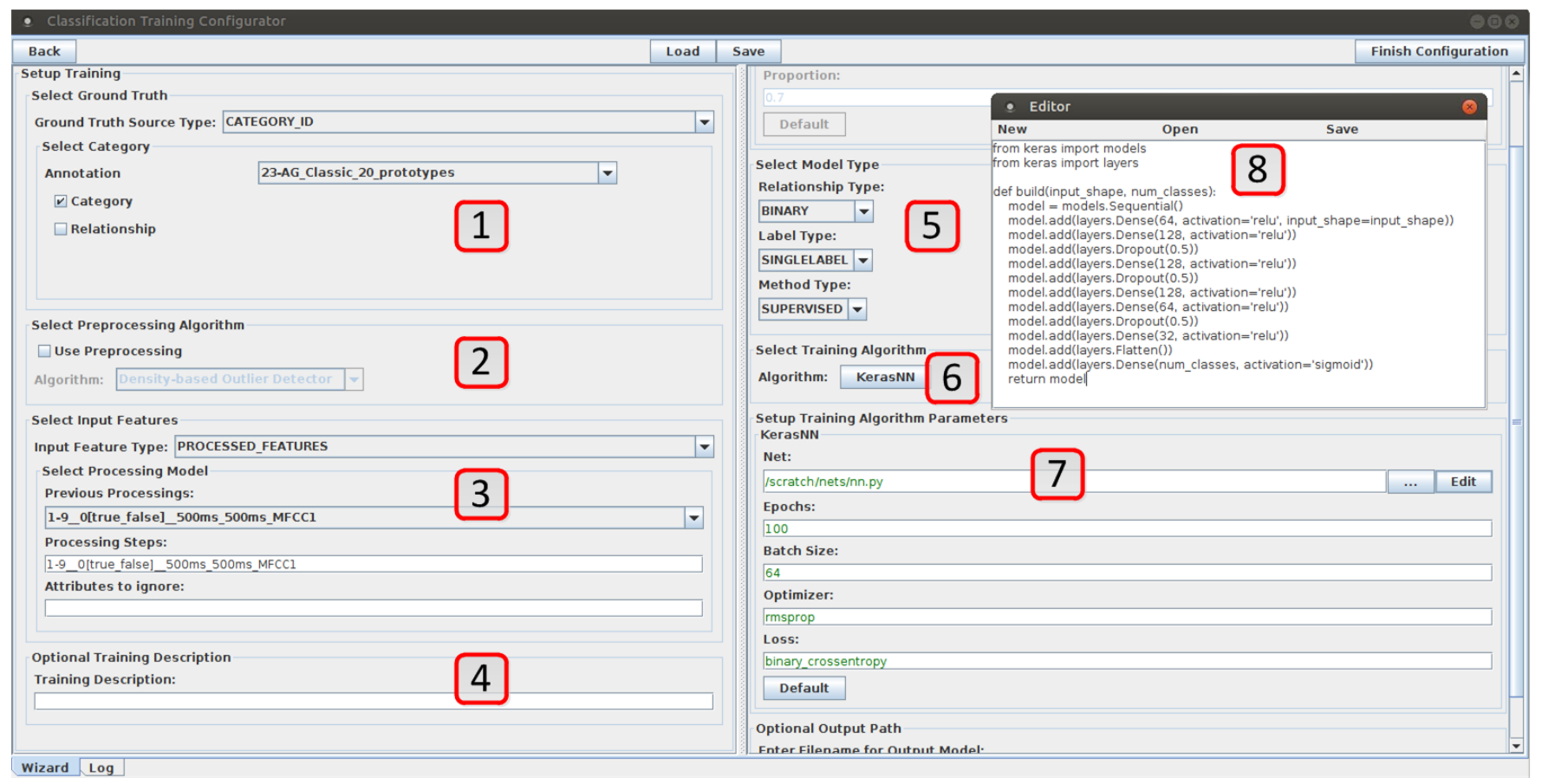

Figure 3: Screenshot of the setup of classification with Keras.

Figure 3 shows a screenshot for the configuration of model training with Keras. The following blocks are marked:

1 : Selection of the ground truth-either as a previously defined category with a fixed ID (which can be created with the annotation editor), an external ARFF file with a list of music files (then, AMUSE will search for processed features in the corresponding folder), or as a ready data set with features and labels.

2 : Configuration of an optional preprocessing method.

3 : Setup of feature processing configuration and aggregation to classification windows.

4 : An optional description of the training configuration for the storage of classification models.

5 : The setup of the model type.

6 : Selection of the classification algorithm (here KerasNN).

7 : Setup of algorithm parameters like the batch size and the number of epochs.

8: A code window which shows the network architecture and can be manually edited. The latter possibility to integrate code as an algorithm parameter can be also used for other algorithms.

\subsection{Validation}

Several measures were added to validate event extraction like onset detection or music segmentation: true positives, false negatives, false positives, F-measure, precision, recall, and deviation. In addition, it is possible to set the tolerance boundary around a predicted event which decides whether the prediction was correct or wrong.

\section{ANNOTATION EDITOR}

In the past, it was not possible to create ground truth annotations in AMUSE with a graphical user interface and it was necessary to edit the related ARFF files manually. With the help of an annotation editor, it is now possible to create annotations in a simple way. It is distinguished between annotations of single and multiple tracks.

The example how the annotation editor can be applied to annotate single tracks is provided in Figure 4.

1 : The spectrogram of the currently played track.

2 : Further tools allow for the zooming into frequency or time space, or the normalization of the current spectrogram.

3 : The annotations are placed below the spectrogram.

4 : Different annotation attributes can be selected from existing ones or defined. It is also possible to load previously extracted events and to use them as annotations or compare visually the events extracted from different algorithms.

\begin{tabular}{l}
5 : Exact positions of the annotations. \\
\hline 6 :
\end{tabular}

In the annotation editor for multiple tracks, categories like genres, emotions, or tempo can be assigned to complete music tracks, see Figure 5.

1 : Selection of music tracks.

2 : The list of the loaded tracks. It is also possible to listen to them.

3 : One or more annotations can be added to individual tracks or to the selection of several tracks. 


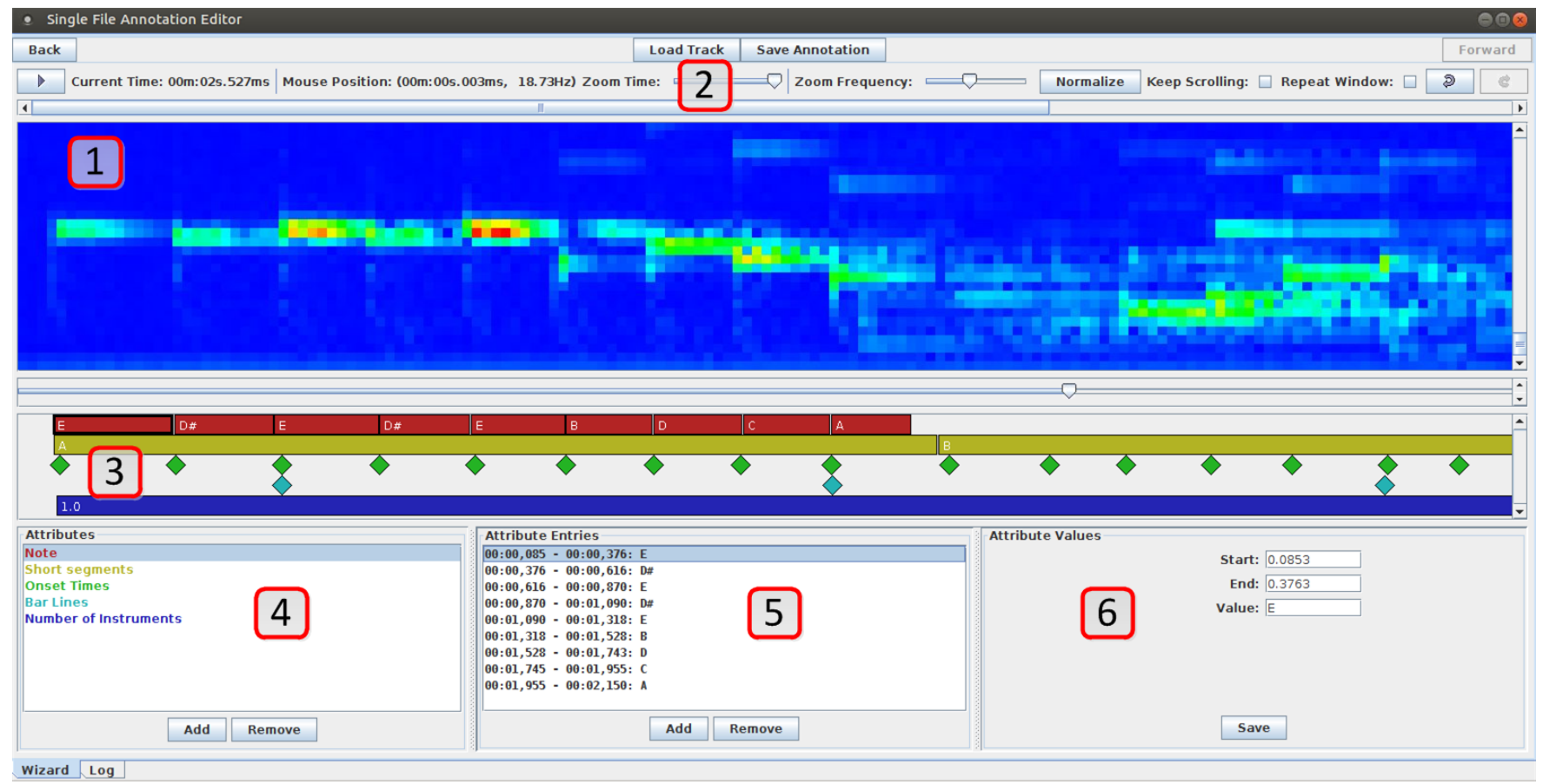

Figure 4: Screenshot of the annotation editor for single tracks.

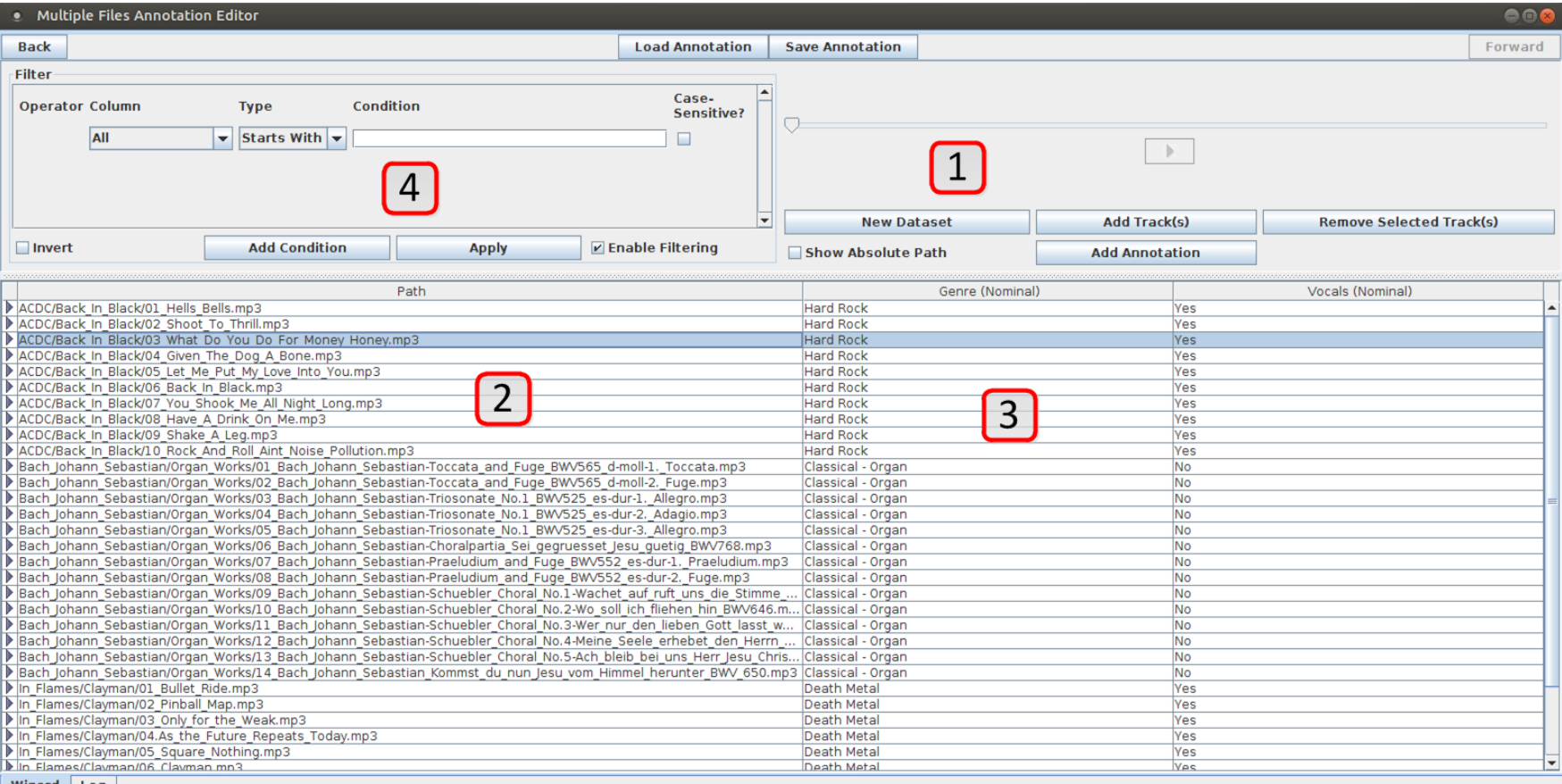

Figure 5: Screenshot of the annotation editor for multiple tracks.

4 : Several filters help to select a subset of loaded tracks, e.g., of a given artist, album, or based on the given pattern in the track name ("Starts with", "Ends with", "Contains", "Is").

\section{FURTHER UPDATES}

In the following, we list several further advancements and improvements. A complete list of all changes is maintained on the GitHub 
webpage. The repository commits are marked either as BUGFIX, UPDATE, NEWFEATURE, or EPIC (for substantial advancements like the first implementation of the annotation editor).

For some music data analysis tasks, in particular beyond the scope of (supervised) classification, the related algorithms may not directly belong to basic AMUSE tasks/nodes like feature extraction, training, etc. (see Figure 1), or it may be desired to avoid restrictions to inputs and outputs defined for AMUSE basic tasks (e.g., that a model applier always stores a model into the corresponding AMUSE workspace folder). For this situation, a new "tool" node was created which allows for the start of flexible tasks. The ARFF file to configure these tasks contains the following attributes:

- ToolClass: Java class which starts the algorithm.

- ToolFolder: folder with further algorithm files, which should be placed in [AMUSEHOME]/[tools].

- ToolConfiguration: configuration file for algorithm settings. The format is free, AMUSE itself has routines to load and save ARFF, Java property, and XML files.

- DestinationFolder: folder for any possible results or logs of the algorithm.

As an example for a "tool" task, the evolutionary approximation of polyphonic audio signals based on mixtures of individual instrument samples was implemented for [19]. Here, the output was the similarity of onsets in music tracks to instrument classes and pitches. Theoretically, this task can be characterized as feature extraction. However, a more flexible handling as a "tool" allowed for the setup of an exhaustive log output describing the progress of evolutionary optimization during the approximation.

Further changes include the possibility to redirect the output of external software like MATLAB or Python modules to AMUSE logger and the serialization of large data sets for a faster saving and loading. In case some parts of an experiment could not be executed (e.g., features could not be processed for a single file from a large number of tracks), AMUSE does not stop but continues the task and provides details to failed parts after the end of the experiment. Individual AMUSE .jar files can be now quickly generated with an ANT script. The handling of batch jobs was updated, and AMUSE supports the current Simple Linux Utility for Resource Management (SLURM) job scheduling system [7], allowing for a parallel execution of different tasks.

\section{RESEARCH AND TEACHING EXPERIENCES}

Several research studies with different goals were conducted with the help of AMUSE. Genre and style recognition was applied, for instance, in [5, 23]. Instrument recognition was considered, among other publications, in [20,22], and vocal recognition in [24]. Music segmentation was optimized in [21]. Evolutionary approximation of instrument texture for genre recognition was introduced in [19].

AMUSE was successfully applied not only for many conference and journal studies, but also for student theses and teaching projects.

In a cooperation teaching project with Loria, Nancy, carried out during the winter term 2018/19, the students have tested how AMUSE can be extended with an unsupervised classification method and a support of Keras. In the bachelor software project "music informatics" which took place in winter terms 2019/20 and 2020/21 at
TU Dortmund University, Department of Computer Science, small groups of students applied AMUSE to learn how signal features can be extracted, configured, and used for music genre recognition. Furthermore, they created simple music recommendation systems and gathered experiences with the application of deep neural networks for music classification. The programming of music genre recognition and music recommendation systems was organized as challenges, in which the students had to accomplish the both tasks within a limited time, and the results were evaluated by the automatic measurement of classification performance for the first task and the manual rating for the second task.

The general outcomes of the application for teaching were twofold. Generally, students enjoyed the projects, and with AMUSE it was possible to gather quick experiences in various tasks related to music data analysis without preliminary courses and deeper backgrounds on music theory, audio signal processing, or machine learning. On the other side, being developed mainly for research studies, the project still had some issues like complex configuration routines and not always comprehensible Java exception messages, which were challenging for students. Many issues were solved after the feedback provided by the course participants, and we continue to make AMUSE more user friendly and plan to integrate it in further student courses.

\section{CONCLUSIONS AND FUTURE WORK}

In this paper, we have presented an overview of advancements and changes over the last decade in AMUSE (Advanced MUSic Explorer)-the open-source Java framework for different tasks in music information retrieval and music data analysis.

The most important changes include the possibility to create ground truth for single and multiple tracks using the annotation editor with a graphical user interface, to apply neural networks with the help of the Keras plugin, to extract more audio features using the Librosa plugin and several other extensions, and to configure feature extraction, processing, and classification in a more flexible way. AMUSE was successfully applied for many research studies with respect to different music classification tasks including genre and emotion recognition, instrument and vocal detection, music segmentation, but also for teaching projects.

There exist many possibilities to extend the framework in future, not only integrating new algorithms for different subtasks, but also providing a more user friendly interface, for instance, presenting the validation results as figures. One particularly promising direction is to integrate modalities (sources of music data) beyond the audio signal, as multi-modal approaches bear great opportunities for music classification and analysis [12, 17, 18]. Because AMUSE is an open-source framework, we hope that the community beyond researchers and students at TU Dortmund University will contribute to further extensions and updates.

\section{ACKNOWLEDGEMENTS}

This work was partly funded by the DFG (German Research Foundation, project 336599081 "Evolutionary optimisation for interpretable music segmentation and music categorisation based on discretised semantic metafeatures"). 


\section{REFERENCES}

[1] C. Cannam, M. O. Jewell, C. Rhodes, M. Sandler, and M. d'Inverno. 2010. Linked Data And You: Bringing music research software into the Semantic Web. Journal of New Music Research 39, 4 (2010), 313-325.

[2] F. Chollet et al. 2021. Keras. https://keras.io. Accessed on: 01.03.2021.

[3] E. Frank, M. A. Hall, G. Holmes, R. Kirkby, and B. Pfahringer. 2005. WEKA - A Machine Learning Workbench for Data Mining. In The Data Mining and Knowledge Discovery Handbook, Oded Maimon and Lior Rokach (Eds.). Springer 1305-1314.

[4] E. Frank, M. A. Hall, and I. H. Witten. 2016. The WEKA Workbench. Online Appendix for "Data Mining: Practical Machine Learning Tools and Techniques" (4 ed.). Morgan Kaufmann.

[5] P. Ginsel, I. Vatolkin, and G. Rudolph. 2020. Analysis of Structural Complexity Features for Music Genre Recognition. In Proceedings of the IEEE Congress on Evolutionary Computation (CEC). IEEE, 1-8. https://doi.org/10.1109/CEC48606.2 020.9185540

[6] M. Hofmann and R. Klinkenberg. 2013. RapidMiner: Data Mining Use Cases and Business Analytics Applications. Chapman \& Hall/CRC.

[7] M. A. Jette, A. B. Yoo, and M. Grondona. 2002. SLURM: Simple Linux Utility for Resource Management. In In Lecture Notes in Computer Science: Proceedings of Job Scheduling Strategies for Parallel Processing (7SSPP). Springer-Verlag, 44-60.

[8] O. Lartillot, P. Toiviainen, and T. Eerola. 2008. A Matlab Toolbox for Music Information Retrieval. In Data Analysis, Machine Learning and Applications, C. Preisach, H. Burkhardt, L. Schmidt-Thieme, and R. Decker (Eds.). Springer, Berlin, Heidelberg, 261-268.

[9] M. Mauch and M. Levy. 2011. Structural Change on Multiple Time Scales as a Correlate of Musical Complexity. In Proceedings of the 12th International Society for Music Information Retrieval Conference (ISMIR), A. Klapuri and C. Leider (Eds.) University of Miami, 489-494.

[10] D. McEnnis, C. McKay, I. Fujinaga, and P. Depalle. 2005. jAudio: An Feature Extraction Library. In Proceedings of the 6th International Conference on Music Information Retrieval (ISMIR). 600-603.

[11] B. McFee, C. Raffel, D. Liang, D. P. W. Ellis, M. McVicar, E. Battenberg, and O. Nieto. 2015. Librosa: Audio and Music Signal Analysis in Python. In Proceedings the Python Science Conference. 18-25.

[12] C. McKay, J. A. Burgoyne, J. Hockman, J. B. L. Smith, G. Vigliensoni, and I. Fujinaga. 2010. Evaluating the Genre Classification Performance of Lyrical Features Relative to Audio, Symbolic and Cultural Features. In Proceedings of the 11th International Society for Music Information Retrieval Conference (ISMIR). 213-218.

[13] I. Mierswa, M. Wurst, R. Klinkenberg, M. Scholz, and T. Euler. 2006. YALE: Rapid Prototyping for Complex Data Mining Tasks. In Proceedings of the 12th ACM SIGKDD International Conference on Knowledge Discovery and Data Mining (KDD), T. Eliassi-Rad, L. H. Ungar, M. Craven, and D. Gunopulos (Eds.). ACM, 935-940.
[14] M. Müller and S. Ewert. 2010. Towards Timbre-Invariant Audio Features for Harmony-Based Music. IEEE Transactions on Audio, Speech, and Language Processing 18, 3 (2010), 649-662.

[15] M. Müller and S. Ewert. 2011. Chroma Toolbox: Matlab Implementations for Extracting Variants of Chroma-Based Audio Features. In Proceedings of the 12th International Society for Music Information Retrieval Conference (ISMIR), A. Klapuri and C. Leider (Eds.). University of Miami, 215-220.

[16] M. Müller and F. Zalkow. 2019. FMP Notebooks: Educational Material for Teaching and Learning Fundamentals of Music Processing. In Proceedings of the 20th International Conference on Music Information Retrieval (ISMIR). 573-580.

[17] S. Oramas, F. Barbieri, O. Nieto, and X. Serra. 2018. Multimodal Deep Learning for Music Genre Classification. Transactions of the International Society for Music Information Retrieval 1, 1 (2018), 4-21.

[18] F. Simonetta, S. Ntalampiras, and F. Avanzini. 2019. Multimodal Music Information Processing and Retrieval: Survey and Future Challenges. In Proceedings of the 2019 International Workshop on Multilayer Music Representation and Processing $(M M R P)$. IEEE, $10-18$

[19] I. Vatolkin. 2020. Evolutionary Approximation of Instrumental Texture in Polyphonic Audio Recordings. In Proceedings of the IEEE Congress on Evolutionary Computation (CEC). IEEE, 1-8. https://doi.org/10.1109/CEC48606.2020.9185506

[20] I. Vatolkin, B. Adrian, and J. Kuzmic. 2021. A Fusion of Deep and Shallow Learning to Predict Genres Based on Instrument and Timbre Features. In Accepted for Proceedings of the 10th International Conference on Artificial Intelligence in Music, Sound, Art and Design.

[21] I. Vatolkin, M. Koch, and M. Müller. 2021. A Multi-Objective Evolutionary Approach to Identify Relevant Audio Features for Music Segmentation. In Accepted for Proceedings of the 10th International Conference on Artificial Intelligence in Music, Sound, Art and Design.

[22] I. Vatolkin, M. Preuß, G. Rudolph, M. Eichhoff, and C. Weihs. 2012. Multi-Objective Evolutionary Feature Selection for Instrument Recognition in Polyphonic Audio Mixtures. Soft Computing 16, 12 (2012), 2027-2047. https://doi.org/10.1007/s005 00-012-0874-9

[23] I. Vatolkin, G. Rudolph, and C. Weihs. 2015. Evaluation of Album Effect for Feature Selection in Music Genre Recognition. In Proceedings of the 16th International Society for Music Information Retrieval Conference (ISMIR). 169-175.

[24] I. Vatolkin and D. Stoller. 2019. Evolutionary Multi-objective Training Set Selection of Data Instances and Augmentations for Vocal Detection. In Proceedings of the 8th International Conference on Computational Intelligence in Music, Sound, Art and Design (EvoMUSART) (Lecture Notes in Computer Science, Vol. 11453). Springer, 201-216. https://doi.org/10.1007/978-3-030-16667-0_14

[25] I. Vatolkin, W. Theimer, and M. Botteck. 2010. AMUSE (Advanced MUSic Explorer) - A Multitool Framework for Music Data Analysis. In Proceedings of the 11th International Society on Music Information Retrieval Conference (ISMIR), J. S. Downie and R. C. Veltkamp (Eds.). 33-38.

[26] L. A. Zadeh. 1965. Fuzzy Sets. Information and Control 8, 3 (1965), 338-353. 